\title{
Motor abilities: methods of strength and strength endurance development in middle-school-aged boys in a 4-week physical training cycle
}

\author{
Veremeenko V.Yu. ${ }^{A B C D E}$, Khudolii O.M. ${ }^{A B C D E}$, Ivashchenko O.V. ${ }^{A B C D E}$ \\ Faculty of Physical Education and Sports, H.S. Skovoroda Kharkiv National Pedagogical University, Ukraine
}

Authors' Contribution: A - Study design; B - Data collection; C - Statistical analysis; D - Manuscript Preparation; E - Funds Collection

\begin{abstract}
Purpose: $\quad$ The study purpose was to develop a technology of programming for strength and strength endurance development in middle-school-aged boys in a 4-week physical training cycle.

Material: $\quad$ The study participants were boys of $6^{\text {th }}$ grade $(n=35), 7^{\text {th }}$ grade $(n=36), 8^{\text {th }}$ grade $(n=36)$. The study materials were processed by the IBM SPSS 23 statistical analysis software. The following parameters were calculated: arithmetic mean (X); standard deviation (s). The probability of difference in statistical indicators was estimated using the Student's t-test.

Results: $\quad$ The analysis of the study results demonstrated that after using a combined method of strength development (1-6 classes), the experimental group boys showed a statistically significant improvement in results in the set of tests $(p<0.05)$. After using a combined method of strength development, the $6^{\text {th }}-8^{\text {th }}$ grade boys had the largest increase in the results of strength and strength endurance of shoulder muscles, strength and strength endurance of abdominal muscles, strength and static strength endurance of leg muscles $(p<0.05)$. After using a circuit training method (7-12 classes), the experimental group boys also showed a statistically significant improvement in results in the set of tests $(p<0.05)$. The $6^{\text {th }}-8^{\text {th }}$ grade boys had the largest increase in the results of dynamic and static strength endurance of shoulder muscles, abdominal and back muscles, leg muscles $(p<0.05)$. According to the Eurofit motor fitness tests, after using the combined method of strength development (1-6 classes) and the circuit training method (7-12 classes) in a 4-week training cycle, the experimental group boys showed a statistically significant improvement in results in the set of tests $(p<$ 0.05). During the experiment, the $6^{\text {th }}-8^{\text {th }}$ grade boys of the control group showed no statistically significant improvement in test results for most parameters $(p>0.05)$. A comparison between the levels of strength fitness of the control group boys and experimental group boys after the experiment showed that the experimental group boys had statistically significantly better results of strength and strength endurance of shoulder muscles, abdominal and back muscles, leg muscles $(\mathrm{p}<0.05)$.

Conclusions: $\quad$ After using the combined method of strength development (1-6 classes) and the circuit training method (7-12 classes) in a 4-week physical training cycle, the middle-school-aged boys showed positive dynamics of strength and strength endurance development of shoulder muscles, abdominal and back muscles, leg muscles. The dynamics of strength and static endurance of the local muscle group is strongly influenced by the combined method of strength development. The circuit training method is effective to develop dynamic and static strength endurance of the local muscle group. For overall development of strength and general endurance, it is effective to use a combination of the combined method of strength development and circuit training method in a 4-week physical training cycle.

Keywords: boys, circuit training method, combined method, mischool age.
\end{abstract}

\section{Introduction}

Studying motor fitness of schoolchildren is one of the most important issues in school physical education [1, $2,3]$. A solution to this issue is related to the following perspectives:

- $\quad$ optimization of motor activity, health promotion and protection of children and adolescents [1, 4];

- $\quad$ planning and control of motor abilities development $[5,6]$;

- $\quad$ improvement in teaching physical exercises $[7,8]$.

The papers by Liakh [9], Ivaschenko [5] focus on the search for means and methods aimed at developing motor abilities in children and adolescents. The researchers determined the principles of strength development methods, revealed regularities, means and methods, as well as pedagogical control over motor abilities development (c) VeremeenkoV.Yu., Khudolii O.M., Ivashchenko O.V., 2019 doi:10.15561/18189172.2019.0208 in schoolchildren $[9,5]$, identified the influence of various forms of lessons on improving motor fitness of middleschool-aged boys, found that boys of this age undergo an overall development of motor abilities $[10,11]$.

The analysis of scientific literature showed that:

- $\quad$ strength fitness can be regarded as a basis for speed and endurance development $[12,11]$;

- circuit training method is effective for developing general and local strength endurance $[12,13]$;

- combined method of strength development is preferable for overall development of muscle groups [14].

Thus, additional research is needed to study the impact of a series of physical training classes using the combined method of strength development and the circuit training method on the dynamics of strength abilities and strength endurance development in middle-school-aged boys. 
The study purpose was to develop methods for strength and strength endurance development in middle-schoolaged boys in a 4-week physical training cycle.

The study object was the process of physical education of $6^{\text {th }}-8^{\text {th }}$ grade boys.

\section{Materials and methods}

Participants. The study participants were boys of $6^{\text {th }}$ grade $(n=35), 7^{\text {th }}$ grade $(n=36), 8^{\text {th }}$ grade $(n=36)$. The children and their parents were fully informed about all the features of the study and gave their consent to participate in the experiment.

Research Design. The study was conducted in a 4-week physical training cycle. For experimental groups, lessons in developing strength and strength endurance of shoulder muscles, abdominal and back muscles, leg muscles were conducted according to the following scheme: combined method of strength development, 1-6 classes; mode of exercising: dynamic effort method, 5 repetitions with a 45-second rest interval between sets; maximum effort method, 3 repetitions with a 45 -second rest interval between sets; isometric effort method, 3 repetitions with a 45-second rest interval; repetition effort method, 10-12 repetitions with a 45 -second rest interval; circuit training method, 7-12 classes.

The circuit training included two classes aimed at developing shoulder muscle endurance (first - maximum, second $-50 \%$ of maximum), two classes developing abdominal and back muscle endurance (first - maximum, second $-50 \%$ of maximum), two classes developing leg muscle endurance (first - maximum, second $-50 \%$ of maximum).

For control groups, an overall development of motor abilities was carried out according to the curriculum during physical training classes.

To solve the tasks set, the following research methods were used: analysis of scientific and methodological literature, pedagogical testing, and methods of mathematical statistics for processing research results.

The experimental group was tested before the experiment, after six classes of combined strength development and after six circuit training classes. The control group was tested before the experiment and after a 4-week cycle according to the school curriculum.

Testing procedure. The testing procedure included commonly known tests $[9,5,16]$.

Test 1. Pull-Up / Chin Up Test (low crossbar), quantity of times;

Test 2. Bent Arm Hang Test (two hands), sec.;

Test 3. Pull-Up / Chin Up Test (Rope Climbing), quantity of times;

Test 4. Cadence Push-Up Test, quantity of times;

Test 5 . The subject lies in prone position, arms bent at the elbow 90 degrees - hold position in seconds;

Test 6. Pull Up Bar - Straight Leg Hanging Leg Raises, quantity of times;

Test 7. Hanging Leg Raises, sec.;

Test 8 . Decline Reverse Crunch on Bench, quantity of times;
Test 9. Trunk Lift Test, quantity of times;

Test 10. Squats Test (two legs), quantity of times;

Test 11. Single Leg Squat (SLS) Test - right leg, quantity of times;

Test 12. Single Leg Squat (SLS) Test - left leg, quantity of times;

Test 13. Single Leg Squat (Pistol) - right leg;

Test 14. Single Leg Squat (Pistol) - left leg;

Test 15. Handgrip Strength Test, kg;

Test 16. Standing Long Jump Test (Broad Jump), cm;

Test 17. Eurofit Sit Up Test (for $30 \mathrm{sec}$.), quantity of times;

Test 18. 4x9 m Shuttle Run Test, sec.;

Test 19. Hand Tapping Test, sec.;

Test 20. Seated Forward Bend, cm;

Test 21. Flamingo Balance Test - single leg balance test;

Test 22. Harvard Step Test.

During shoulder muscle testing, the following parameters were measured: Pull-Up / Chin Up Test (shoulder flexion strength), Bent Arm Hang Test (shoulder flexion static endurance), Pull-Up / Chin Up Test (Rope Climbing) (shoulder flexion strength endurance), Cadence Push-Up Test and The subject lies in prone position, arms bent at the elbow 90 degrees - hold position in seconds (shoulder extension strength).

During abdominal and back muscle testing, the following parameters were measured: Pull Up Bar Straight Leg Hanging Leg Raises (abdominal muscle strength), Hanging Leg Raises (abdominal muscle static endurance), Decline Reverse Crunch on Bench (abdominal muscle strength endurance), Trunk Lift Test (back muscle endurance).

During leg muscle testing, the following parameters were measured: Squats Test (two legs) (leg muscle strength endurance), Single Leg Squat Test - right leg, Single Leg Squat Test - left leg (leg muscle strength), Single Leg Squat (Pistol) - right leg, Single Leg Squat (Pistol) - left leg (leg muscle static endurance).

The study also measured motor abilities parameters using the Eurofit tests: Handgrip Strength Test (hand flexion strength), Standing Long Jump Test (speed and strength), Eurofit Sit Up Test (for $30 \mathrm{sec}$.) (abdominal muscle strength endurance), 4x9 m Shuttle Run Test (dexterity), Hand Tapping Test (speed), Seated Forward Bend (flexibility), Flamingo Balance Test (static balance of the body).

Statistical analysis. The study used the IBM SPSS 23 software. For each variable, the following statistics were calculated: mean values, standard deviations, Student's t-test for paired samples and Student's t-test for independent samples.

The study protocol was approved by the Ethical Committee of H. S. Skovoroda Kharkiv National Pedagogical University. In addition, the children and their parents or legal guardians were fully informed about all the features of the study, and a signed informed-consent document was obtained from all the parents. 
Results

Tables 1-6 present the test results of strength fitness of the control and experimental group boys.

After the combined method of strength development (see Table 1), the experimental group boys showed a statistically significant improvement in results in the set of tests $(\mathrm{p}<0.05)$

The test results of shoulder muscle strength of the $6^{\text {th }}$

Table 1. Test results of strength fitness of $6^{\text {th }}-8^{\text {th }}$ grade boys (experimental group). Combined method of strength development

\begin{tabular}{|c|c|c|c|c|c|c|c|c|c|c|}
\hline \multirow{2}{*}{ No. } & \multirow{2}{*}{ Test } & \multirow{2}{*}{ Grade } & \multicolumn{2}{|c|}{ Before experiment } & \multicolumn{2}{|c|}{ After experiment } & \multirow[t]{2}{*}{ Increase } & \multirow[b]{2}{*}{$\%$} & \multirow{2}{*}{$\mathbf{t}$} & \multirow{2}{*}{$\mathbf{P}$} \\
\hline & & & $\mathbf{x}$ & s & $\mathbf{x}$ & s & & & & \\
\hline \multirow{3}{*}{1} & \multirow{3}{*}{$\begin{array}{l}\text { Pull-Up / Chin Up Test } \\
\text { (low crossbar), quantity } \\
\text { of times }\end{array}$} & 6 & 3.368 & 1.257 & 4.053 & 1.026 & -0.684 & 20.3 & -4.444 & 0.000 \\
\hline & & 7 & 4.790 & 1.932 & 5.316 & 1.701 & -0.526 & 11.0 & -3.750 & 0.001 \\
\hline & & 8 & 5.895 & 2.558 & 6.316 & 2.311 & -0.421 & 7.1 & -3.618 & 0.002 \\
\hline \multirow{3}{*}{2} & \multirow{3}{*}{$\begin{array}{l}\text { Bent Arm Hang Test } \\
\text { (two hands), sec. }\end{array}$} & 6 & 4.226 & 1.276 & 4.700 & 0.946 & -0.474 & 11.2 & -3.911 & 0.001 \\
\hline & & 7 & 5.758 & 1.987 & 6.337 & 1.817 & -0.579 & 10.0 & -9.977 & 0.000 \\
\hline & & 8 & 6.626 & 1.918 & 7.000 & 1.842 & -0.374 & 5.6 & -8.400 & 0.000 \\
\hline \multirow{3}{*}{3} & Pull-Up / Chin Up & 6 & 9.579 & 2.775 & 10.526 & 2.480 & -0.947 & 9.9 & -5.295 & 0.000 \\
\hline & \multirow{2}{*}{$\begin{array}{l}\text { Test (Rope Climbing), } \\
\text { quantity of times }\end{array}$} & 7 & 10.737 & 2.705 & 11.895 & 2.664 & -1.158 & 10.8 & -8.382 & 0.000 \\
\hline & & 8 & 13.368 & 2.910 & 14.474 & 2.776 & -1.105 & 8.3 & -10.500 & 0.000 \\
\hline \multirow{3}{*}{4} & \multirow{3}{*}{$\begin{array}{l}\text { Cadence Push-Up Test, } \\
\text { quantity of times }\end{array}$} & 6 & 17.895 & 3.213 & 19.263 & 3.494 & -1.368 & 7.6 & -6.245 & 0.000 \\
\hline & & 7 & 18.842 & 4.598 & 20.158 & 4.259 & -1.316 & 7.0 & -6.994 & 0.000 \\
\hline & & 8 & 21.684 & 3.575 & 22.632 & 3.113 & -0.947 & 4.4 & -4.025 & 0.001 \\
\hline \multirow{3}{*}{5} & \multirow{3}{*}{$\begin{array}{l}\text { The subject lies in } \\
\text { prone position, arms } \\
\text { bent at the elbow } 90 \\
\text { degrees - hold position } \\
\text { in seconds }\end{array}$} & 6 & 13.016 & 2.661 & 13.321 & 2.637 & -0.305 & 2.3 & -8.420 & 0.000 \\
\hline & & 7 & 14.116 & 2.904 & 14.542 & 2.926 & -0.426 & 3.0 & -9.583 & 0.000 \\
\hline & & 8 & 17.168 & 2.077 & 17.411 & 2.064 & -0.242 & 1.4 & -7.608 & 0.000 \\
\hline \multirow{3}{*}{6} & Pull Up Bar- Straight & 6 & 4.316 & 0.820 & 4.842 & 0.834 & -0.526 & 12.2 & -4.472 & 0.000 \\
\hline & \multirow{3}{*}{$\begin{array}{l}\text { Leg Hanging Leg Raises, } \\
\text { quantity of times }\end{array}$} & 7 & 5.526 & 1.073 & 6.158 & 0.958 & -0.632 & 11.4 & -5.555 & 0.000 \\
\hline & & 8 & 6.263 & 0.991 & 6.842 & 0.765 & -0.579 & 9.2 & -4.158 & 0.001 \\
\hline \multirow{3}{*}{7} & & 6 & 5.247 & 0.646 & 5.689 & 0.624 & -0.442 & 8.4 & -8.232 & 0.000 \\
\hline & Hanging Leg Raises, sec. & 7 & 6.179 & 0.991 & 6.574 & 0.982 & -0.395 & 6.4 & -8.791 & 0.000 \\
\hline & & 8 & 7.321 & 0.961 & 7.637 & 0.941 & -0.316 & 4.3 & -9.409 & 0.000 \\
\hline & Decline Reverse Crunch & 6 & 9.947 & 1.870 & 10.684 & 1.827 & -0.737 & 7.4 & -4.916 & 0.000 \\
\hline 8 & on Bench, quantity of & 7 & 9.632 & 1.892 & 10.737 & 2.104 & -1.105 & 11.5 & -6.533 & 0.000 \\
\hline & & 8 & 14.105 & 2.706 & 15.105 & 2.331 & -1.000 & 7.1 & -7.550 & 0.000 \\
\hline & & 6 & 24.737 & 2.257 & 26.158 & 1.979 & -1.421 & 5.7 & -5.092 & 0.000 \\
\hline 9 & of times & 7 & 26.316 & 2.382 & 26.895 & 2.052 & -0.579 & 2.2 & -3.012 & 0.007 \\
\hline & & 8 & 29.947 & 3.045 & 30.474 & 2.855 & -0.526 & 1.8 & -3.750 & 0.001 \\
\hline & & 6 & 27.263 & 3.445 & 29.263 & 3.142 & -2.000 & 7.3 & -7.886 & 0.000 \\
\hline 10 & Squats Tes & 7 & 28.263 & 4.331 & 29.789 & 3.489 & -1.526 & 5.4 & -5.459 & 0.000 \\
\hline & & 8 & 33.632 & 3.004 & 34.842 & 2.363 & -1.211 & 3.6 & -5.750 & 0.000 \\
\hline & Single Leg Squat (SLS) & 6 & 2.211 & 0.855 & 2.368 & 0.761 & -0.158 & 7.1 & -1.837 & 0.083 \\
\hline 11 & Test - right leg, quantity & 7 & 2.842 & 1.167 & 3.000 & 1.000 & -0.158 & 5.6 & -1.837 & 0.083 \\
\hline & of times & 8 & 3.105 & 0.936 & 3.211 & 0.855 & -0.105 & 3.4 & -1.455 & 0.163 \\
\hline & Single Leg Squat (SLS) & 6 & 1.842 & 0.834 & 1.895 & 0.809 & -0.053 & 2.9 & -1.000 & 0.331 \\
\hline 12 & Test - left leg, quantity & 7 & 2.158 & 1.068 & 2.263 & 0.991 & -0.105 & 4.9 & -1.455 & 0.163 \\
\hline & of times & 8 & 2.579 & 0.961 & 2.684 & 0.885 & -0.105 & 4.1 & -1.455 & 0.163 \\
\hline & & 6 & 4.684 & 0.825 & 5.279 & 0.766 & -0.595 & 12.7 & -9.012 & 0.000 \\
\hline 13 & g squat (PIStOI) & 7 & 5.005 & 1.315 & 5.474 & 1.232 & -0.468 & 9.4 & -9.930 & 0.000 \\
\hline & & 8 & 5.705 & 1.083 & 6.295 & 0.977 & -0.589 & 10.3 & -14.098 & 0.000 \\
\hline & & 6 & 4.211 & 0.836 & 4.732 & 0.827 & -0.521 & 12.4 & -9.984 & 0.000 \\
\hline 14 & SIngle Leg squat (PIstol) & 7 & 4.311 & 1.176 & 4.826 & 1.218 & -0.516 & 12.0 & -11.875 & 0.000 \\
\hline & & 8 & 5.232 & 0.972 & 5.595 & 1.031 & -0.363 & 6.9 & -6.986 & 0.000 \\
\hline
\end{tabular}


Table 2. Test results of strength fitness of $6^{\text {th }}-8^{\text {th }}$ grade boys (experimental group). Circuit training method

\begin{tabular}{|c|c|c|c|c|c|c|c|c|c|c|}
\hline \multirow[t]{2}{*}{ No. } & \multirow[t]{2}{*}{ Test } & \multirow[t]{2}{*}{ Grade } & \multicolumn{2}{|c|}{$\begin{array}{l}\text { Before } \\
\text { experiment }\end{array}$} & \multicolumn{2}{|c|}{ After experiment } & \multirow[t]{2}{*}{ Increase } & \multirow[b]{2}{*}{$\%$} & \multirow[t]{2}{*}{$\mathbf{t}$} & \multirow[t]{2}{*}{$\mathbf{P}$} \\
\hline & & & $\mathbf{x}$ & $\mathbf{s}$ & $\mathbf{x}$ & $\mathbf{s}$ & & & & \\
\hline \multirow{3}{*}{1} & \multirow{3}{*}{$\begin{array}{l}\text { Pull-Up / Chin Up Test } \\
\text { (low crossbar), quantity } \\
\text { of times }\end{array}$} & 6 & 4.053 & 1.026 & 4.368 & 0.895 & -0.316 & 7.8 & -2.882 & 0.010 \\
\hline & & 7 & 5.316 & 1.701 & 5.684 & 1.416 & -0.368 & 6.9 & -2.348 & 0.031 \\
\hline & & 8 & 6.316 & 2.311 & 6.789 & 1.988 & -0.474 & 7.5 & -4.025 & 0.001 \\
\hline \multirow{3}{*}{2} & \multirow{3}{*}{$\begin{array}{l}\text { Bent Arm Hang Test (two } \\
\text { hands), sec. }\end{array}$} & 6 & 4.700 & 0.946 & 5.032 & 0.952 & -0.332 & 7.0 & -7.221 & 0.000 \\
\hline & & 7 & 6.337 & 1.817 & 6.595 & 1.645 & -0.258 & 4.1 & -4.534 & 0.000 \\
\hline & & 8 & 7.000 & 1.841 & 7.453 & 1.746 & -0.453 & 6.5 & -10.246 & 0.000 \\
\hline \multirow{3}{*}{3} & \multirow{3}{*}{$\begin{array}{l}\text { Pull-Up / Chin Up Test } \\
\text { (Rope Climbing), quantity } \\
\text { of times }\end{array}$} & 6 & 10.526 & 2.480 & 11.263 & 2.207 & -0.737 & 7.0 & -4.379 & 0.000 \\
\hline & & 7 & 11.895 & 2.664 & 12.474 & 2.366 & -0.579 & 4.9 & -4.158 & 0.001 \\
\hline & & 8 & 14.474 & 2.776 & 15.263 & 2.156 & -0.789 & 5.5 & -3.174 & 0.005 \\
\hline \multirow{3}{*}{4} & \multirow{3}{*}{$\begin{array}{l}\text { Cadence Push-Up Test, } \\
\text { quantity of times }\end{array}$} & 6 & 19.263 & 3.494 & 19.947 & 3.027 & -0.684 & 3.5 & -4.444 & 0.000 \\
\hline & & 7 & 20.158 & 4.259 & 20.947 & 3.865 & -0.789 & 3.9 & -4.371 & 0.000 \\
\hline & & 8 & 22.632 & 3.113 & 23.421 & 2.950 & -0.789 & 3.5 & -4.371 & 0.000 \\
\hline \multirow{3}{*}{5} & \multirow{3}{*}{$\begin{array}{l}\text { The subject lies in prone } \\
\text { position, arms bent at the } \\
\text { elbow } 90 \text { degrees - hold } \\
\text { position in seconds }\end{array}$} & 6 & 13.321 & 2.637 & 13.568 & 2.598 & -0.247 & 1.9 & -7.762 & 0.000 \\
\hline & & 7 & 14.542 & 2.926 & 15.037 & 2.804 & -0.495 & 3.4 & -8.534 & 0.000 \\
\hline & & 8 & 15.105 & 2.331 & 15.842 & 1.803 & -0.737 & 4.9 & -4.379 & 0.000 \\
\hline \multirow{3}{*}{6} & Pull Up Bar- Straight & 6 & 4.842 & 0.834 & 5.053 & 0.780 & -0.211 & 4.4 & -2.191 & 0.042 \\
\hline & \multirow{3}{*}{$\begin{array}{l}\text { Leg Hanging Leg Raises, } \\
\text { quantity of times }\end{array}$} & 7 & 6.158 & 0.958 & 6.632 & 1.116 & -0.474 & 7.7 & -3.375 & 0.003 \\
\hline & & 8 & 6.842 & 0.765 & 7.579 & 1.017 & -0.737 & 10.8 & -4.379 & 0.000 \\
\hline \multirow{3}{*}{7} & & 6 & 5.689 & 0.624 & 5.926 & 0.550 & -0.237 & 4.2 & -5.077 & 0.000 \\
\hline & Hanging Leg Raises, sec. & 7 & 6.574 & 0.982 & 7.026 & 0.902 & -0.453 & 6.9 & -7.618 & 0.000 \\
\hline & & 8 & 7.637 & 0.941 & 8.105 & 0.874 & -0.468 & 6.1 & -8.836 & 0.000 \\
\hline & Decline Reverse Crunch & 6 & 10.684 & 1.827 & 11.789 & 1.813 & -1.105 & 10.3 & -5.144 & 0.000 \\
\hline 8 & on Bench, quantity of & 7 & 10.737 & 2.104 & 11.474 & 2.038 & -0.737 & 6.9 & -4.379 & 0.000 \\
\hline & times & 8 & 15.105 & 2.331 & 15.842 & 1.803 & -0.737 & 4.9 & -4.379 & 0.000 \\
\hline & & 6 & 26.158 & 1.979 & 26.737 & 1.759 & -0.579 & 2.2 & -3.644 & 0.002 \\
\hline 9 & siti lest, quantity & 7 & 26.895 & 2.052 & 27.579 & 1.924 & -0.684 & 2.5 & -3.980 & 0.001 \\
\hline & & 8 & 30.474 & 2.855 & 31.211 & 2.417 & -0.737 & 2.4 & -3.986 & 0.001 \\
\hline & & 6 & 29.263 & 3.142 & 30.105 & 2.846 & -0.842 & 2.9 & -4.086 & 0.001 \\
\hline 10 & ity of times & 7 & 29.789 & 3.489 & 30.684 & 3.233 & -0.895 & 3.0 & -4.819 & 0.000 \\
\hline & & 8 & 34.842 & 2.363 & 36.211 & 1.686 & -1.368 & 3.9 & -4.083 & 0.001 \\
\hline & Single Leg Squat (SLS) & 6 & 2.368 & 0.761 & 2.421 & 0.692 & -0.053 & 2.2 & -1.000 & 0.331 \\
\hline 11 & Test - right leg, quantity & 7 & 3.000 & 1.000 & 3.105 & 0.936 & -0.105 & 3.5 & -1.455 & 0.163 \\
\hline & of times & 8 & 3.211 & 0.855 & 3.316 & 0.749 & -0.105 & 3.3 & -1.455 & 0.163 \\
\hline & Single Leg Squat (SLS) & 6 & 1.895 & 0.809 & 1.947 & 0.705 & -0.053 & 2.8 & -1.000 & 0.331 \\
\hline 12 & Test - left leg, quantity of & 7 & 2.263 & 0.991 & 2.316 & 0.946 & -0.053 & 2.3 & -1.000 & 0.331 \\
\hline & times & 8 & 2.684 & 0.885 & 2.789 & 0.787 & -0.105 & 3.9 & -1.455 & 0.163 \\
\hline & & 6 & 5.279 & 0.766 & 5.668 & 0.745 & -0.389 & 7.4 & -7.869 & 0.000 \\
\hline 13 & single Leg squat (PIStoI) - & 7 & 5.474 & 1.232 & 6.105 & 1.166 & -0.632 & 11.5 & -8.298 & 0.000 \\
\hline & & 8 & 6.295 & 0.977 & 6.784 & 0.828 & -0.489 & 7.8 & 10.398 & 0.000 \\
\hline & & 6 & 4.732 & 0.827 & 5.158 & 0.726 & -0.426 & 9.0 & -6.162 & 0.000 \\
\hline 14 & single Leg squat (PIStol) - & 7 & 4.826 & 1.218 & 5.274 & 1.228 & -0.447 & 9.3 & -8.591 & 0.000 \\
\hline & & 8 & 5.595 & 1.031 & 6.105 & 0.890 & -0.511 & 9.1 & -8.224 & 0.000 \\
\hline
\end{tabular}

grade boys statistically significantly increased in test 1 (shoulder flexion strength) by $20.3 \%$; in test 2 (shoulder flexion static endurance) by $11.2 \%$; in test 3 (shoulder flexion strength endurance) by $9.9 \%$; in test 4 (shoulder extension strength) by $7.6 \%$. The test results of abdominal and back muscle strength statistically significantly increased in test 6 (abdominal muscle strength) by $12.2 \%$; in test 7 (abdominal muscle static endurance) by $8.4 \%$; in test 8 (abdominal muscle strength endurance) by $7.4 \%$. The test results of leg muscle strength statistically significantly increased in test 10 (leg muscle strength endurance) by $7.3 \%$; in tests 13 and 14 (leg muscle static 
Table 3. Test results of strength fitness of $6^{\text {th }}-8^{\text {th }}$ grade boys (control group). Circuit training method

\begin{tabular}{|c|c|c|c|c|c|c|c|c|c|c|c|}
\hline \multirow[t]{2}{*}{ No. } & \multirow[t]{2}{*}{ Test } & \multirow[t]{2}{*}{ Grade } & \multirow[t]{2}{*}{$\mathbf{n}$} & \multicolumn{2}{|c|}{$\begin{array}{l}\text { Before } \\
\text { experiment }\end{array}$} & \multicolumn{2}{|c|}{$\begin{array}{l}\text { After } \\
\text { experiment }\end{array}$} & \multirow[t]{2}{*}{ Increase } & \multirow[b]{2}{*}{$\%$} & \multirow[t]{2}{*}{$\mathbf{t}$} & \multirow[t]{2}{*}{$\mathbf{P}$} \\
\hline & & & & $\mathbf{x}$ & $s$ & $x$ & $\mathbf{s}$ & & & & \\
\hline \multirow{3}{*}{1} & \multirow{3}{*}{$\begin{array}{l}\text { Pull-Up / Chin Up Test (low } \\
\text { crossbar), quantity of times }\end{array}$} & 6 & 16 & 3.375 & 1.310 & 3.563 & 1.153 & -0.188 & 5.6 & -1.861 & 0.083 \\
\hline & & 7 & 17 & 4.706 & 2.469 & 4.824 & 2.351 & -0.118 & 2.5 & -1.461 & 0.163 \\
\hline & & 8 & 17 & 5.941 & 2.657 & 6.059 & 2.633 & -0.118 & 2.0 & -1.461 & 0.163 \\
\hline \multirow{3}{*}{2} & \multirow{3}{*}{$\begin{array}{l}\text { Bent Arm Hang Test (two } \\
\text { hands), sec. }\end{array}$} & 6 & 16 & 4.225 & 1.205 & 4.319 & 1.228 & -0.094 & 2.2 & -1.996 & 0.064 \\
\hline & & 7 & 17 & 4.965 & 1.879 & 5.059 & 1.771 & -0.094 & 1.9 & -1.793 & 0.092 \\
\hline & & 8 & 17 & 5.976 & 1.816 & 6.018 & 1.797 & -0.041 & 0.7 & -1.692 & 0.110 \\
\hline \multirow{3}{*}{3} & \multirow{3}{*}{$\begin{array}{l}\text { Pull-Up / Chin Up Test (Rope } \\
\text { Climbing), quantity of times }\end{array}$} & 6 & 16 & 10.063 & 3.193 & 10.250 & 3.109 & -0.188 & 1.9 & -1.861 & 0.083 \\
\hline & & 7 & 17 & 9.235 & 2.927 & 9.353 & 2.871 & -0.118 & 1.3 & -1.461 & 0.163 \\
\hline & & 8 & 17 & 13.000 & 2.718 & 13.235 & 2.562 & -0.235 & 1.8 & -2.219 & 0.041 \\
\hline \multirow{3}{*}{4} & \multirow{3}{*}{$\begin{array}{l}\text { Cadence Push-Up Test, } \\
\text { quantity of times }\end{array}$} & 6 & 16 & 18.625 & 3.344 & 18.750 & 3.256 & -0.125 & 0.7 & -1.464 & 0.164 \\
\hline & & 7 & 17 & 18.176 & 4.640 & 18.294 & 4.469 & -0.118 & 0.6 & -1.461 & 0.163 \\
\hline & & 8 & 17 & 20.941 & 3.363 & 21.118 & 3.219 & -0.176 & 0.8 & -1.852 & 0.083 \\
\hline \multirow{3}{*}{5} & \multirow{3}{*}{$\begin{array}{l}\text { The subject lies in prone } \\
\text { position, arms bent at the } \\
\text { elbow } 90 \text { degrees - hold } \\
\text { position in seconds }\end{array}$} & 6 & 16 & 13.587 & 2.273 & 13.700 & 2.192 & -0.113 & 0.8 & -1.840 & 0.086 \\
\hline & & 7 & 17 & 14.329 & 2.665 & 14.447 & 2.688 & -0.118 & 0.8 & -2.311 & 0.034 \\
\hline & & 8 & 17 & 16.618 & 2.282 & 16.612 & 2.252 & 0.006 & 0.0 & 0.120 & 0.906 \\
\hline \multirow{3}{*}{6} & Pull Up Bar- Straight Leg & 6 & 16 & 4.063 & 1.124 & 4.188 & 1.047 & -0.125 & 3.0 & -1.464 & 0.164 \\
\hline & Hanging Leg Raises, quantity & 7 & 17 & 5.471 & 1.375 & 5.588 & 1.326 & -0.117 & 2.1 & -1.461 & 0.163 \\
\hline & of times & 8 & 17 & 7.000 & 1.275 & 7.118 & 1.111 & -0.118 & 1.7 & -1.461 & 0.163 \\
\hline & & 6 & 16 & 4.856 & 0.904 & 4.900 & 0.878 & -0.044 & 0.9 & -1.282 & 0.219 \\
\hline 7 & Hanging Leg Raises, sec. & 7 & 17 & 5.994 & 0.957 & 6.018 & 0.927 & -0.023 & 0.4 & -0.746 & 0.466 \\
\hline & & 8 & 17 & 7.141 & 0.879 & 7.118 & 0.821 & 0.023 & 0.3 & 0.523 & 0.608 \\
\hline & & 6 & 16 & 10.125 & 2.579 & 10.250 & 2.569 & -0.125 & 1.2 & -1.464 & 0.164 \\
\hline 8 & $B$ & 7 & 17 & 9.882 & 2.088 & 10.059 & 2.045 & -0.176 & 1.8 & -1.852 & 0.083 \\
\hline & & 8 & 17 & 13.353 & 2.805 & 13.471 & 2.672 & -0.118 & 0.9 & -1.461 & 0.163 \\
\hline & & 6 & 16 & 23.563 & 2.632 & 23.687 & 2.469 & -0.125 & 0.5 & -1.464 & 0.164 \\
\hline 9 & & 7 & 17 & 23.765 & 3.052 & 23.941 & 2.839 & -0.176 & 0.7 & -1.852 & 0.083 \\
\hline & & 8 & 17 & 27.471 & 3.281 & 27.647 & 3.121 & -0.176 & 0.6 & -1.852 & 0.083 \\
\hline & & 6 & 16 & 27.937 & 3.193 & 28.063 & 3.065 & -0.125 & 0.4 & -1.464 & 0.164 \\
\hline 10 & & 7 & 17 & 28.294 & 4.606 & 28.647 & 4.242 & -0.353 & 1.2 & -2.073 & 0.055 \\
\hline & & 8 & 17 & 31.823 & 2.481 & 32.000 & 2.424 & -0.176 & 0.5 & -1.376 & 0.188 \\
\hline & & 6 & 16 & 1.813 & 0.911 & 1.813 & 0.911 & - & - & - & - \\
\hline 11 & of times & 7 & 17 & 2.353 & 1.057 & 2.353 & 1.057 & - & - & - & - \\
\hline & & 8 & 17 & 2.647 & 1.057 & 2.647 & 1.057 & - & - & - & - \\
\hline & & 6 & 16 & 1.563 & 0.727 & 1.563 & 0.727 & - & - & - & - \\
\hline 12 & 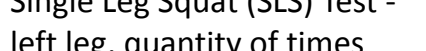 & 7 & 17 & 1.941 & 0.899 & 1.941 & 0.899 & - & - & - & - \\
\hline & & 8 & 17 & 2.471 & 0.874 & 2.471 & 0.874 & - & - & - & - \\
\hline & & 6 & 16 & 4.431 & 0.973 & 4.469 & 0.982 & -0.038 & 0.8 & -1.861 & 0.083 \\
\hline 13 & $g$ & 7 & 17 & 4.188 & 1.252 & 4.318 & 1.155 & -0.129 & 3.0 & -2.637 & 0.018 \\
\hline & & 8 & 17 & 4.918 & 1.172 & 4.988 & 1.118 & -0.070 & 1.4 & -1.509 & 0.151 \\
\hline & & 6 & 16 & 3.950 & 0.848 & 3.994 & 0.854 & -0.044 & 1.1 & -1.447 & 0.168 \\
\hline 14 & gite Leg squat (tistol) - ieil & 7 & 17 & 3.735 & 1.127 & 3.806 & 1.057 & -0.071 & 1.9 & -1.900 & 0.076 \\
\hline & & 8 & 17 & 4.782 & 1.197 & 4.818 & 1.199 & -0.035 & 0.7 & -1.144 & 0.269 \\
\hline
\end{tabular}

endurance) by $12.7 \%$ and $12.4 \%$, respectively.

The test results of shoulder muscle strength of the $7^{\text {th }}$ grade experimental group boys statistically significantly increased in test 1 (shoulder flexion strength) by $11.0 \%$; in test 2 (shoulder flexion static endurance) by $10.0 \%$ and in test 3 (shoulder flexion strength endurance) by $10.8 \%$.
The test results of abdominal and back muscle strength statistically significantly increased in test 6 (abdominal muscle strength) by $11.4 \%$ and in test 8 (abdominal muscle strength endurance) by $11.5 \%(\mathrm{p}<0.05)$. The test results of leg muscle strength statistically significantly increased in test 10 (leg muscle strength endurance) by 
Table 4. Test results of strength fitness of $6^{\text {th }}-8^{\text {th }}$ grade boys after a one-month physical training cycle using the combined method of strength development and the circuit training method (experimental group).

\begin{tabular}{|c|c|c|c|c|c|c|c|c|c|c|c|}
\hline \multirow[t]{2}{*}{ No. } & \multirow[t]{2}{*}{ Test } & \multirow[t]{2}{*}{ Grade } & \multirow[t]{2}{*}{ n } & \multicolumn{2}{|c|}{$\begin{array}{l}\text { Before } \\
\text { experiment }\end{array}$} & \multicolumn{2}{|c|}{$\begin{array}{l}\text { After } \\
\text { experiment }\end{array}$} & \multicolumn{2}{|c|}{ Increase } & \multirow[t]{2}{*}{$t$} & \multirow[t]{2}{*}{$\mathbf{P}$} \\
\hline & & & & $\mathbf{x}$ & $\mathbf{s}$ & $\mathbf{x}$ & $\mathbf{s}$ & & $\%$ & & \\
\hline \multirow{3}{*}{15} & \multirow{3}{*}{$\begin{array}{l}\text { Handgrip Strength Test, } \\
\text { kg }\end{array}$} & 6 & 19 & 16.305 & 1.407 & 16.421 & 1.387 & -0.116 & 0.7 & -3.450 & 0.003 \\
\hline & & 7 & 19 & 17.579 & 1.458 & 17.868 & 1.416 & -0.289 & 1.6 & -8.090 & 0.000 \\
\hline & & 8 & 19 & 18.147 & 1.916 & 18.374 & 1.855 & -0.226 & 1.2 & -5.712 & 0.000 \\
\hline \multirow{3}{*}{16} & \multirow{3}{*}{$\begin{array}{l}\text { Standing Long Jump Test } \\
\text { (Broad Jump), cm }\end{array}$} & 6 & 19 & 1.542 & 0.133 & 1.546 & 0.127 & -0.004 & 0.3 & -1.455 & 0.163 \\
\hline & & 7 & 19 & 1.589 & 0.210 & 1.611 & 0.199 & -0.022 & 1.4 & -4.686 & 0.000 \\
\hline & & 8 & 19 & 1.671 & 0.213 & 1.685 & 0.205 & -0.015 & 0.9 & -3.441 & 0.003 \\
\hline \multirow{3}{*}{17} & \multirow{3}{*}{$\begin{array}{l}\text { Eurofit Sit Up Test (for } 30 \\
\text { sec.), quantity of times }\end{array}$} & 6 & 19 & 23.947 & 3.613 & 24.579 & 3.024 & -0.632 & 2.6 & -2.721 & 0.014 \\
\hline & & 7 & 19 & 26.684 & 4.888 & 27.789 & 4.144 & -1.105 & 4.1 & -4.025 & 0.001 \\
\hline & & 8 & 19 & 26.263 & 3.413 & 27.158 & 2.814 & -0.895 & 3.4 & -3.923 & 0.001 \\
\hline \multirow{3}{*}{18} & \multirow{3}{*}{$\begin{array}{l}4 \times 9 \text { m Shuttle Run Test, } \\
\text { sec. }\end{array}$} & 6 & 19 & 11.616 & 0.462 & 11.463 & 0.469 & 0.153 & 1.3 & 4.422 & 0.000 \\
\hline & & 7 & 19 & 11.374 & 0.605 & 11.263 & 0.576 & 0.111 & 1.0 & 3.625 & 0.002 \\
\hline & & 8 & 19 & 11.037 & 0.680 & 10.926 & 0.689 & 0.111 & 1.0 & 3.745 & 0.001 \\
\hline \multirow{3}{*}{19} & \multirow{4}{*}{ Hand Tapping Test, sec. } & 6 & 19 & 13.937 & 0.779 & 13.779 & 0.755 & 0.158 & 1.1 & 5.276 & 0.000 \\
\hline & & 7 & 19 & 13.632 & 0.987 & 13.484 & 0.944 & 0.147 & 1.0 & 4.169 & 0.001 \\
\hline & & 8 & 19 & 12.263 & 0.617 & 12.168 & 0.576 & 0.095 & 0.8 & 3.375 & 0.003 \\
\hline \multirow{3}{*}{20} & & 6 & 19 & 5.895 & 1.100 & 5.947 & 0.911 & -0.053 & 0.9 & -0.438 & 0.667 \\
\hline & \multirow{2}{*}{ Seated Forward Bend, cm } & 7 & 19 & 5.474 & 1.172 & 5.368 & 0.895 & 0.105 & 2.7 & 1.000 & 0.331 \\
\hline & & 8 & 19 & 4.526 & 1.429 & 4.474 & 1.219 & 0.053 & 1.2 & 0.567 & 0.578 \\
\hline \multirow{4}{*}{21} & \multirow{3}{*}{$\begin{array}{l}\text { Flamingo Balance test - } \\
\text { single leg balance test }\end{array}$} & 6 & 19 & 9.000 & 1.856 & 8.789 & 1.475 & 0.211 & 2.3 & 1.455 & 0.163 \\
\hline & & 7 & 19 & 7.895 & 1.449 & 7.895 & 1.243 & 0.000 & 0.0 & 0.000 & 1.000 \\
\hline & & 8 & 19 & 8.105 & 1.595 & 8.158 & 1.259 & -0.053 & 0.7 & -0.325 & 0.749 \\
\hline & & 6 & 19 & 63.421 & 4.046 & 65.000 & 3.756 & -1.579 & 2.5 & -4.962 & 0.000 \\
\hline \multirow[t]{2}{*}{22} & Harvard Step Test & 7 & 19 & 65.684 & 5.508 & 67.632 & 6.112 & -1.947 & 3.0 & -4.401 & 0.000 \\
\hline & & 8 & 19 & 67.421 & 5.337 & 68.737 & 5.636 & -1.316 & 2.0 & -3.664 & 0.002 \\
\hline
\end{tabular}

Table 5. Test results of motor fitness of the control group boys after a 4-week physical training cycle according to the school curriculum

\begin{tabular}{|c|c|c|c|c|c|c|c|c|c|c|c|}
\hline \multirow[t]{2}{*}{ No. } & \multirow[t]{2}{*}{ Test } & \multirow[t]{2}{*}{ Grade } & \multirow[t]{2}{*}{$\mathbf{n}$} & \multicolumn{2}{|c|}{$\begin{array}{l}\text { Before } \\
\text { experiment }\end{array}$} & \multicolumn{2}{|c|}{$\begin{array}{l}\text { After } \\
\text { experiment }\end{array}$} & \multicolumn{2}{|c|}{ Increase } & \multirow[t]{2}{*}{$\mathbf{t}$} & \multirow[t]{2}{*}{$\mathbf{P}$} \\
\hline & & & & $\mathrm{x}$ & $\mathbf{s}$ & $\mathbf{x}$ & s & & $\%$ & & \\
\hline \multirow{3}{*}{15} & \multirow{3}{*}{ Handgrip Strength Test, kg } & 6 & 16 & 16.419 & 1.313 & 16.456 & 1.327 & -0.038 & 0.2 & -1.695 & 0.111 \\
\hline & & 7 & 17 & 16.012 & 1.796 & 16.059 & 1.784 & -0.047 & 0.3 & -2.057 & 0.056 \\
\hline & & 8 & 17 & 16.900 & 1.569 & 16.923 & 1.534 & -0.024 & 0.1 & -0.497 & 0.626 \\
\hline \multirow{3}{*}{16} & \multirow{3}{*}{$\begin{array}{l}\text { Standing Long Jump Test } \\
\text { (Broad Jump), cm }\end{array}$} & 6 & 16 & 1.540 & 0.125 & 1.541 & 0.125 & -0.001 & 0.1 & -1.000 & 0.333 \\
\hline & & 7 & 17 & 1.574 & 0.173 & 1.574 & 0.170 & -0.000 & 0.0 & -0.270 & 0.791 \\
\hline & & 8 & 17 & 1.705 & 0.143 & 1.705 & 0.143 & 0.000 & 0.0 & 0.270 & 0.791 \\
\hline \multirow{3}{*}{17} & \multirow{3}{*}{$\begin{array}{l}\text { Eurofit Sit Up Test (for } 30 \\
\text { sec.), quantity of times }\end{array}$} & 6 & 16 & 24.625 & 3.202 & 24.813 & 2.949 & -0.187 & 0.7 & -1.379 & 0.188 \\
\hline & & 7 & 17 & 26.412 & 4.287 & 26.706 & 3.949 & -0.294 & 1.1 & -2.063 & 0.056 \\
\hline & & 8 & 17 & 25.294 & 3.653 & 25.412 & 3.572 & -0.118 & 0.5 & -1.461 & 0.163 \\
\hline \multirow{3}{*}{18} & \multirow{3}{*}{ 4x9 m Shuttle Run Test, sec. } & 6 & 16 & 11.469 & 0.535 & 11.469 & 0.545 & 0.000 & 0.0 & 0.000 & 1.000 \\
\hline & & 7 & 17 & 11.247 & 0.565 & 11.247 & 0.540 & 0.000 & 0.0 & 0.000 & 1.000 \\
\hline & & 8 & 17 & 10.935 & 0.625 & 10.994 & 0.678 & -0.059 & 0.5 & -1.429 & 0.172 \\
\hline \multirow{3}{*}{19} & \multirow{3}{*}{ Hand Tapping Test, sec. } & 6 & 16 & 14.344 & 0.697 & 14.325 & 0.736 & 0.019 & 0.1 & 0.426 & 0.676 \\
\hline & & 7 & 17 & 14.106 & 1.112 & 14.112 & 1.159 & -0.006 & 0.1 & -0.148 & 0.884 \\
\hline & & 8 & 17 & 12.929 & 1.236 & 12.912 & 1.265 & 0.018 & 0.1 & 0.337 & 0.740 \\
\hline \multirow{3}{*}{20} & \multirow{3}{*}{ Seated Forward Bend, cm } & 6 & 16 & 5.813 & & 5.875 & 1.088 & -0.063 & 1.0 & -1.000 & 0.333 \\
\hline & & 7 & 17 & 5.353 & 1.835 & 5.353 & 1.835 & - & - & - & - \\
\hline & & 8 & 17 & 4.941 & 1.435 & 4.941 & 1.435 & - & - & - & - \\
\hline \multirow{3}{*}{21} & \multirow{3}{*}{$\begin{array}{l}\text { Flamingo Balance test - } \\
\text { single leg balance test }\end{array}$} & 6 & 16 & 9.500 & 1.592 & 9.250 & 1.390 & 0.250 & 2.6 & 1.732 & 0.104 \\
\hline & & 7 & 17 & 8.176 & 1.629 & 8.118 & 1.536 & 0.059 & 0.7 & 0.566 & 0.579 \\
\hline & & 8 & 17 & 7.941 & 1.819 & 7.882 & 1.576 & 0.059 & 0.7 & 0.566 & 0.579 \\
\hline \multirow{3}{*}{22} & \multirow{3}{*}{ Harvard Step Test } & 6 & 16 & 66.563 & 3.915 & 66.687 & 3.516 & -0.125 & 0.2 & -0.620 & 0.544 \\
\hline & & 7 & 17 & 71.588 & 5.917 & 71.529 & 5.680 & 0.059 & 0.1 & 0.324 & 0.750 \\
\hline & & 8 & 17 & 68.412 & 4.691 & 68.353 & 5.049 & 0.059 & 0.1 & 0.324 & 0.750 \\
\hline
\end{tabular}


Table 6. Comparative analysis between the levels of strength fitness of the $6^{\text {th }}-8^{\text {th }}$ grade boys of the experimental and control groups after the experiment

\begin{tabular}{|c|c|c|c|c|c|c|c|c|c|}
\hline \multirow{2}{*}{ No } & \multirow{2}{*}{ Test } & \multirow[b]{2}{*}{ Grade } & \multirow[b]{2}{*}{$\mathbf{n}$} & \multicolumn{2}{|c|}{ Experimental group } & \multirow{2}{*}{$\mathbf{n}$} & \multicolumn{2}{|c|}{ Control group } & \multirow{2}{*}{$\mathbf{P}$} \\
\hline & & & & $\mathbf{x}$ & & & $\mathbf{x}$ & & \\
\hline \multirow{3}{*}{1} & Pull-Up / Chin Un Test (low & 6 & 19 & 4.368 & 0.895 & 16 & 3.563 & 1.153 & 0.026 \\
\hline & ruil-up / Crim up rest flow & 7 & 19 & 5.684 & 1.416 & 17 & 4.824 & 2.351 & 0.187 \\
\hline & crossbar), quantity of times & 8 & 19 & 6.789 & 1.988 & 17 & 6.059 & 2.633 & 0.351 \\
\hline \multirow{3}{*}{2} & Bent Arm Hang Test (two hands), & 6 & 19 & 5.032 & 0.952 & 16 & 4.319 & 1.228 & 0.062 \\
\hline & Bent Arm Hang lest (two nanas), & 7 & 19 & 6.595 & 1.645 & 17 & 5.059 & 1.771 & 0.011 \\
\hline & sec. & 8 & 19 & 7.453 & 1.746 & 17 & 6.018 & 1.797 & 0.021 \\
\hline & Pull-Up / Chin Up Test (Rope & 6 & 19 & 11.263 & 2.207 & 16 & 10.250 & 3.109 & 0.269 \\
\hline 3 & Climhing) guantity of times & 7 & 19 & 12.474 & 2.366 & 17 & 9.353 & 2.871 & 0.001 \\
\hline & Climbing), quantity of times & 8 & 19 & 15.263 & 2.156 & 17 & 13.235 & 2.562 & 0.014 \\
\hline & Cadence Push-Up Test, quantity & 6 & 19 & 19.947 & 3.027 & 16 & 18.750 & 3.256 & 0.268 \\
\hline 4 & of times & 7 & 19 & 20.947 & 3.865 & 17 & 18.294 & 4.469 & 0.065 \\
\hline & & 8 & 19 & 23.421 & 2.950 & 17 & 21.118 & 3.219 & 0.032 \\
\hline & The subject lies in prone & 6 & 19 & 13.568 & 2.598 & 16 & 13.700 & 2.192 & 0.874 \\
\hline 5 & position, arms bent at the elbow & 7 & 19 & 15.037 & 2.804 & 17 & 14.447 & 2.688 & 0.525 \\
\hline & $\begin{array}{l}90 \text { degrees - hold position in } \\
\text { seconds }\end{array}$ & 8 & 19 & 17.758 & 2.051 & 17 & 16.612 & 2.252 & 0.119 \\
\hline & Pull Up Bar- Straight Leg Hanging & 6 & 19 & 5.053 & 0.780 & 16 & 4.188 & 1.047 & 0.008 \\
\hline 6 & Leg Raises, quantity of times & 7 & 19 & 6.632 & 1.116 & 17 & 5.588 & 1.326 & 0.015 \\
\hline & & 8 & 19 & 7.579 & 1.017 & 17 & 7.118 & 1.111 & 0.202 \\
\hline & & 6 & 19 & 5.926 & 0.550 & 16 & 4.900 & 0.878 & 0.000 \\
\hline 7 & Hanging Leg Raises, sec. & 7 & 19 & 7.026 & 0.902 & 17 & 6.018 & 0.927 & 0.002 \\
\hline & & 8 & 19 & 8.105 & 0.874 & 17 & 7.118 & 0.821 & 0.001 \\
\hline & Decline Reverse Crunch on & 6 & 19 & 11.789 & 1.813 & 16 & 10.250 & 2.569 & 0.046 \\
\hline 8 & Ronch & 7 & 19 & 11.474 & 2.038 & 17 & 10.059 & 2.045 & 0.046 \\
\hline & Bench, quantity of times & 8 & 19 & 15.842 & 1.803 & 17 & 13.471 & 2.672 & 0.003 \\
\hline & & 6 & 19 & 26.737 & 1.759 & 16 & 23.687 & 2.469 & 0.000 \\
\hline 9 & Trunk Lift Test, quantity of times & 7 & 19 & 27.579 & 1.924 & 17 & 23.941 & 2.839 & 0.000 \\
\hline & & 8 & 19 & 31.211 & 2.417 & 17 & 27.647 & 3.121 & 0.000 \\
\hline & & 6 & 19 & 30.105 & 2.846 & 16 & 28.063 & 3.065 & 0.049 \\
\hline 10 & squats lest (two legs), quantity & 7 & 19 & 30.684 & 3.233 & 17 & 28.647 & 4.242 & 0.112 \\
\hline & of times & 8 & 19 & 36.211 & 1.686 & 17 & 32.000 & 2.424 & 0.000 \\
\hline & Single Leg Squat (SLS) Test - right & 6 & 19 & 2.421 & 0.692 & 16 & 1.813 & 0.911 & 0.032 \\
\hline 11 & 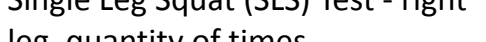 & 7 & 19 & 3.105 & 0.936 & 17 & 2.353 & 1.057 & 0.030 \\
\hline & & 8 & 19 & 3.316 & 0.749 & 17 & 2.647 & 1.057 & 0.034 \\
\hline & Single Leg Squat (SLS) Test - left & 6 & 19 & 1.947 & 0.705 & 16 & 1.563 & 0.727 & 0.122 \\
\hline 12 & single Leg squat (SLD) lest - Ien & 7 & 19 & 2.316 & 0.946 & 17 & 1.941 & 0.899 & 0.233 \\
\hline & leg, quantity of times & 8 & 19 & 2.789 & 0.787 & 17 & 2.471 & 0.874 & 0.258 \\
\hline & Single Leg Squat (Pistol) - right & 6 & 19 & 5.668 & 0.745 & 16 & 4.469 & 0.982 & 0.000 \\
\hline 13 & & 7 & 19 & 6.105 & 1.166 & 17 & 4.318 & 1.155 & 0.000 \\
\hline & & 8 & 19 & 6.784 & 0.828 & 17 & 4.988 & 1.118 & 0.000 \\
\hline & & 6 & 19 & 5.158 & 0.726 & 16 & 3.994 & 0.854 & 0.000 \\
\hline 14 & Single Leg Squat (Pistol) - left leg & 7 & 19 & 5.274 & 1.228 & 17 & 3.806 & 1.057 & 0.001 \\
\hline & & 8 & 19 & 6.105 & 0.890 & 17 & 4.818 & 1.199 & 0.001 \\
\hline & & 6 & 19 & 16.421 & 1.387 & 16 & 16.456 & 1.327 & 0.940 \\
\hline 15 & Handgrip Strength Test, kg & 7 & 19 & 17.868 & 1.416 & 17 & 16.059 & 1.784 & 0.002 \\
\hline & & 8 & 19 & 18.374 & 1.855 & 17 & 16.923 & 1.534 & 0.016 \\
\hline & Standing Long Jump Test (Broad & 6 & 19 & 1.546 & 0.127 & 16 & 1.541 & 0.125 & 0.905 \\
\hline 16 & stanalng Long Jump lest (Broad & 7 & 19 & 1.611 & 0.199 & 17 & 1.574 & 0.170 & 0.562 \\
\hline & Jump), cm & 8 & 19 & 1.685 & 0.205 & 17 & 1.705 & 0.143 & 0.746 \\
\hline & Eurofit Sit Up Test (for $30 \mathrm{sec}$.) & 6 & 19 & 1.546 & 0.127 & 16 & 1.541 & 0.125 & 0.905 \\
\hline 17 & curo & 7 & 19 & 27.789 & 4.144 & 17 & 26.706 & 3.949 & 0.429 \\
\hline & & 8 & 19 & 27.158 & 2.814 & 17 & 25.412 & 3.572 & 0.111 \\
\hline & & 6 & 19 & 11.463 & 0.469 & 16 & 11.469 & 0.545 & 0.974 \\
\hline 18 & 4x9 m Shuttle Run Test, sec. & 7 & 19 & 11.263 & 0.576 & 17 & 11.247 & 0.540 & 0.932 \\
\hline & & 8 & 19 & 10.926 & 0.689 & 17 & 10.994 & 0.678 & 0.768 \\
\hline & & 6 & 19 & 13.779 & 0.755 & 16 & 14.325 & 0.736 & 0.038 \\
\hline 19 & Hand Tapping Test, sec. & 7 & 19 & 13.484 & 0.944 & 17 & 14.112 & 1.159 & 0.083 \\
\hline & & 8 & 19 & 12.168 & 0.576 & 17 & 12.912 & 1.265 & 0.027 \\
\hline & & 6 & 19 & 5.947 & 0.911 & 16 & 5.875 & 1.088 & 0.832 \\
\hline 20 & Seated Forward Bend, cm & 7 & 19 & 5.368 & 0.895 & 17 & 5.353 & 1.835 & 0.974 \\
\hline & & 8 & 19 & 4.474 & 1.219 & 17 & 4.941 & 1.435 & 0.298 \\
\hline & & 6 & 19 & 8.789 & 1.475 & 16 & 9.250 & 1.390 & 0.352 \\
\hline 21 & Flamingo Balance test - single leg & 7 & 19 & 7.895 & 1.243 & 17 & 8.118 & 1.536 & 0.634 \\
\hline & balance test & 8 & 19 & 8.158 & 1.259 & 17 & 7.882 & 1.576 & 0.564 \\
\hline & & 6 & 19 & 65.000 & 3.756 & 16 & 66.687 & 3.516 & 0.182 \\
\hline 22 & Harvard Step Test & 7 & 19 & 67.632 & 6.112 & 17 & 71.529 & 5.680 & 0.056 \\
\hline & & 8 & 19 & 68.737 & 5.636 & 17 & 68.353 & 5.049 & 0.832 \\
\hline
\end{tabular}


$5.4 \%$ and in tests 13 and 14 (leg muscle static endurance) by $9.4 \%$ and $12.0 \%$, respectively $(\mathrm{p}<0.001)$.

The test results of shoulder muscle strength of the $8^{\text {th }}$ grade experimental group boys statistically significantly increased in test 1 (shoulder flexion strength) by $7.1 \%$, test 2 (shoulder flexion static endurance) and test 3 (shoulder flexion strength endurance) by $5.6 \%$ and $8.3 \%$, respectively. The test results of abdominal and back muscle strength statistically significantly increased in test 6 (abdominal muscle strength) by $9.2 \%$ and in test 8 (abdominal muscle strength endurance) by $7.1 \%(\mathrm{p}<$ 0.001 ). The test results of leg muscle strength statistically significantly increased in tests 13 and 14 (leg muscle static endurance) by $10.3 \%$ and $6.9 \%$, respectively.

After the circuit training method (see Table 2), the experimental group boys showed a statistically significant improvement in results in the set of tests $(p<0.05)$.

The test results of shoulder muscle strength of the $6^{\text {th }}$ grade experimental group boys statistically significantly increased in test 1 (shoulder flexion strength) by $7.8 \%$; in test 2 (shoulder flexion static endurance) and test 3 (shoulder flexion strength endurance) by $7.0 \%$ and $7.0 \%$, respectively $(p<0.001)$. The test results of abdominal and back muscle strength statistically significantly increased in test 8 (abdominal muscle strength endurance) by $10.3 \%(p<0.001)$. The test results of leg muscle strength statistically significantly increased in tests 13 and 14 (leg muscle static endurance) by $7.4 \%$ and $9.0 \%$, respectively $(\mathrm{p}<0.001)$.

The test results of shoulder muscle strength of the $7^{\text {th }}$ grade experimental group boys statistically significantly increased in test 1 (shoulder flexion strength) by $6.9 \%$ and in test 3 (shoulder flexion strength endurance) by $4.9 \%$ (p $<0.001$ ). The test results of abdominal and back muscle strength statistically significantly increased in test 6 (abdominal muscle strength) by $7.7 \%$, in test 7 (abdominal muscle static endurance) and 8 (abdominal muscle strength endurance) by $6.9 \%$ and $6.9 \%$, respectively. The test results of leg muscle strength statistically significantly increased in tests 13 and 14 (leg muscle static endurance) by $11.5 \%$ and $9.3 \%$, respectively ( $<<0.001$ ).

The test results of shoulder muscle strength of the $8^{\text {th }}$ grade experimental group boys statistically significantly increased in test 1 (shoulder flexion strength) by $7.5 \%$, in test 2 (shoulder flexion static endurance) by $6.5 \%$ and test 3 (shoulder flexion strength endurance) by $5.5 \%$, respectively.

The test results of abdominal and back muscle strength statistically significantly increased in test 6 (abdominal muscle strength) by $10.8 \%$ and in test 7 (abdominal muscle static endurance) by $6.1 \%$. The test results of leg muscle strength statistically significantly increased in tests 13 and 14 (leg muscle static endurance) by $7.8 \%$ and $9.1 \%$, respectively.

During the experiment, the control group boys (see Table 3) showed no statistically significant improvement in the test results for most parameters $(\mathrm{p}>0.05)$. The $7^{\text {th }}$ grade control group boys showed a tendency to improve the results in test 5 (shoulder extension strength) $(\mathrm{p}<0.05)$, the $8^{\text {th }}$ grade boys - in test 3 (shoulder flexion strength endurance) $(p<0.05)$. In other tests, there were no statistically significant differences between the $6^{\text {th }}-8^{\text {th }}$ grade boys $(\mathrm{p}>0.05)$.

According to the Eurofit motor fitness tests, after a 4-week training cycle, the experimental group boys (see Table 4) showed a statistically significant improvement in results in the set of tests $(\mathrm{p}<0.05)$.

The results of the $6^{\text {th }}$ grade experimental group boys statistically significantly increased in test 17 (abdominal muscle strength endurance) by $2.6 \%$ and in test 22 (Harvard Step Test) by $2.5 \%(\mathrm{p}<0.001)$.

The results of the $7^{\text {th }}$ grade boys statistically significantly increased in test 17 (abdominal muscle strength endurance) by $4.1 \%$ and in test 22 (general endurance) by $3.0 \%(\mathrm{p}<0.001)$.

The results of the $8^{\text {th }}$ grade experimental group boys statistically significantly increased in test 17 (abdominal muscle strength endurance) by $3.4 \%$ and in test 22 (general endurance) by $2.0 \%(\mathrm{p}<0.05)$.

In other tests, the $6^{\text {th }}-8^{\text {th }}$ grade boys showed a tendency to improve the test results $(\mathrm{p}>0.05)$.

During the experiment, the control group boys (see Table 5) showed no statistically significant improvement in the test results for most parameters $(\mathrm{p}>0.05)$.

A comparison between the levels of strength fitness of the control group boys and experimental group boys after the experiment (see Table 6 ) showed that the $6^{\text {th }}$ grade boys of the experimental group had statistically significantly better test results of strength and strength endurance of shoulder muscles, abdominal and back muscles in tests 1 , $6,7,8,9(\mathrm{p}<0.05)$, and statistically significantly better test results of leg muscle strength in tests $10,11,13,14$ $(\mathrm{p}<0.05)$.

A comparison between the levels of strength fitness of the control group boys and experimental group boys after the experiment (see Table 7) showed that the $7^{\text {th }}$ grade boys of the experimental group had statistically significantly better test results of strength and strength endurance of shoulder muscles, abdominal and back muscles in tests $2,3,6,7,8,9(\mathrm{p}<0.05)$, and statistically significantly better test results of leg muscle strength in tests 13,14 (p $<0.05$ ).

A comparison between the levels of strength fitness of the control group boys and experimental group boys after the experiment (see Table 8 ) showed that the $8^{\text {th }}$ grade boys of the experimental group had statistically significantly better test results of strength and strength endurance of shoulder muscles, abdominal and back muscles in tests $2,3,4,5,7,8,9(\mathrm{p}<0.05)$, and statistically significantly better test results of leg muscle strength in tests $10,11,13$, $14(\mathrm{p}<0.05)$.

\section{Discussion}

The study assumed that the use of the combined method of strength development and the circuit training method in a 4-week physical training cycle would positively affect the dynamics of strength and strength endurance development in $6^{\text {th }}-8^{\text {th }}$ grade boys. 
After six classes (1-6) using the combined method of strength development, the $6^{\text {th }}-8^{\text {th }}$ grade boys showed a statistically significant positive dynamics in the results of strength fitness and endurance. The largest increase (by $20.3 \%$ ) was observed in shoulder flexion strength in the $6^{\text {th }}$ grade boys. These data supplement the findings of Cieślicka et al. [10], Ivashchenko et al. [15] on the effectiveness of the combined method of strength development during school physical training classes.

After six classes (7-12) using the circuit training method, the $6^{\text {th }}-8^{\text {th }}$ grade boys showed a statistically significant positive dynamics in the results of strength fitness and endurance. The test results increased by $2-11 \%$. These data confirm the findings of Poperekov et al. [13] on the effectiveness of the circuit training method during physical training of children and adolescents.

The study ascertained that the use of the combined method of strength development and the circuit training method in a one-month physical training cycle positively affected the overall development of motor abilities in the $6^{\text {th }}-8^{\text {th }}$ grade boys. The obtained results of the study characterize the peculiarities of the dynamics of middle schoolers' motor fitness and supplement the data of Ivashchenko et al. [17], James et al. [18] on the holistic character of motor abilities development in children and adolescents; the data of Blagrove et al. [19], Prykhodko [20] on the regularities of motor abilities development in children and adolescents.

Consequently, the study results indicate that the combined method of strength development and the circuit training method in a 4-week physical training cycle have a statistically significant effect on the dynamics of motor fitness of middle-school-aged boys.

Further research is required to study the regularities of development and relationship between muscle strength and endurance of middle-school-aged boys.

\section{Conclusions}

After using the combined method of strength development (1-6 classes) and the circuit training method (7-12 classes) in a 4-week physical training cycle, the middle-school-aged boys showed positive dynamics of strength and strength endurance development of shoulder muscles, abdominal and back muscles, leg muscles. The dynamics of strength and static endurance of the local muscle group is strongly influenced by the combined method of strength development. The circuit training method is effective to develop dynamic and static strength endurance of the local muscle group. For overall development of strength and general endurance, it is effective to use a combination of the combined method of strength development and circuit training method in a 4-week physical training cycle.

\section{Acknowledgment}

The research was carried out according to the theme 13.04 "Modelling of the learning process and development of motor abilities in children and adolescents" (20132014) (state registration number 0113U002102).

\section{Conflict of interest}

The authors state that there is no conflict of interest.

\section{References}

1. BădicuG.PhysicalActivityandHealth-RelatedQualityofLifein Adults from Braşov, Romania. Education Sciences, 2018; 8(2). https://doi.org/10.3390/educsci8020052

2. Balsevich VK. Ontokinesiology of man. Moscow: Theory and practice of physical culture; 2000. (in Russian)

3. Novak D, Podnar H, Emeljanovas A, Marttinen R. Comparison of Fitness Levels between Croatian and Lithuanian Students. Montenegrin Journal of Sports Science and Medicine, 2015; 4(1): 5-11.

4. Krutsevych TYu, Bezverkhnia HV. Recreation in the physical culture of different population groups: teaching. manual. Kiev: Olympic Literature; 2010. (in Ukrainian)

5. Ivashchenko OV. Modelling of physical education students. Kharkiv: OVS; 2016. (in Ukrainian)

6. Emeljanovas A, Mieziene B, Putriute V. The Relationship Between Physical Activity and Content of the Physical Education Classes in 11-12 Years Old Lithuanian Schoolchildren. The Pilot Study. Croatian Journal of Education-Hrvatski Casopis Za Odgoj I Obrazovanje, 2015; 17(1): 93-120.

7. Ivashchenko $\mathrm{O}$, Abdulkhalikova $\mathrm{T}$, Cieślicka $\mathrm{M}$. Effectiveness of Motor Skills Development in 5th-7th Grade Girls at Different Modes of Physical Exercises. Teoriâ Ta Metodika Fìzičnogo Vihovannâ, 2017;17(4):201-7. https://doi.org/10.17309/tmfv.2017.4.1205

8. Ivashchenko O, Khudolii O, Iermakov S, Harkusha S. Physical exercises' mastering level in classification of motor preparedness of 11-13 years old boys. Journal of Physical
Education and Sport, 2017;17(3):1031-6. https://doi. org/10.7752/jpes.2017.03158

9. Liakh VI. Driving abilities of schoolchildren: Fundamentals of theory and methods of development. Moscow: TerraSport; 2000.(in Russian)

10.Cieślicka M, Ivashchenko O. Discriminant analysis method to determine the power of the boys 11-12 year. Journal of Education, Health and Sport, 2016; 6(10): 721-9. https://doi.org/10.5281/zenodo.229911

11. Khudolii OM, Ivashchenko OV. Simulation of the learning process and development of motor abilities in children and adolescents. Kharkiv: OVS; 2014. (in Ukrainian).

12.Khudolii OM. General Fundamentals of Theory and Methodology of Physical Education. Kharkiv: OVS; 2008. (in Ukrainian)

13.Poperekov VS, Buldakova N, Bandakov MP, Zhilina NO, Solgalov VS. Focused Development of Jumping Ability in Young Basketball Players By Means of Circuit Training. Human Sport Medicine, 2018;18(4):103-9. https://doi.org/10.14529/hsm180415

14.Liu C, Chen CS, Ho WH, Fule RJ, Chung PH, Shiang TY. The Effects of Passive Leg Press Training on Jumping Performance, Speed, and Muscle Power. Journal of Strength and Conditioning Research, 2013; 27(6): 1479-86.

15.Ivashchenko O, Cieślicka M. Discriminant analysis in the classification of the preparation strength girls 11-12 year. Journal of Education, Health and Sport, 2016; 6(8):888-97. https://doi.org/10.5281/zenodo.229884

16.Khudolii OM, Ivashchenko OV. Simulation of the learning 
process and development of motor abilities in children and adolescents. Kharkiv: OVS; 2014. (in Ukrainian)

17.Ivashchenko O, Khudolii O, Iermakov S, Lochbaum M, Cieślicka M, Zukow W, Nosko M, Yermakova T. Methodological approaches to pedagogical control of the functional and motorfitness of the girls from 7-9 grades. Journal of Physical Education and Sport, 2017; 17(1): 254- 261. https://doi.org/10.7752/jpes.2017.01038

18.James LP, Haff GG., Kelly VG, Connick MJ, Hoffman BW, Beckman EM. The impact of strength level on adaptations to combined weightlifting, plyometric, and ballistic training. Scandinavian Journal of Medicine \& Science in Sports, 2018; 28(5): 1494-1505. https://doi.org/10.1111/sms.13045

19.Blagrove RC, Howe LP, Cushion EJ, Spence A, Howatson G, Pedlar CR, Hayes PR. Effects of Strength Training on Postpubertal Adolescent Distance Runners. Medicine and Science in Sports and Exercise, 2018; 50(6): 1224-1232. https://doi.org/10.1249/mss.0000000000001543

20.Prykhodko VV. The Factor Structure of Coordination Abilities Development in 5th-7th Grade Boys. Teoria Ta Metodika Fìzičnogo Vihovannâ, 2017; 17(4): 191-200. (in Ukrainian) https://doi.org/10.17309/tmfv.2017.4.1204

\section{Information about the authors:}

Veremeenko V. Yu. ; http://orcid.org/0000-0002-9826-9678; viktoriaveremeenko91@gmail.com; Department of Theory and Methodology of Physical Education, Health and Medical Physical Culture, H. S. Skovoroda Kharkiv National Pedagogical University; Alchevskikh St, 29, Kharkiv, 61002, Ukraine.

Khudolii O. M. : (Corresponding author); http://orcid.org/0000-0002-5605-9939; khudolii.oleg@gmail.com; Department of Theory and Methodology of Physical Education, Health and Medical Physical Culture, H. S. Skovoroda Kharkiv National Pedagogical University; Alchevskikh St, 29, Kharkiv, 61003, Ukraine.

Ivashchenko 0. V. ; http://orcid.org/0000-0002-2708-5636; olga@tmfv.com.ua; Department of Theory and Methodology of Physical Education, Health and Medical Physical Culture, H. S. Skovoroda Kharkiv National Pedagogical University; Alchevskikh St, 29, Kharkiv, 61002, Ukraine.

Cite this article as:

Veremeenko VYu, Khudolii OM, Ivashchenko OV. Motor abilities: methods of strength and strength endurance development in middle-school-aged boys in a 4-week physical training cycle. Pedagogics, psychology, medical-biological problems of physical training and sports, 2019;23(2):102-111. https://doi.org/10.15561/18189172.2019.0208

The electronic version of this article is the complete one and can be found online at: https://www.sportpedagogy.org.ua/index.php/PPS/issue/archive

This is an Open Access article distributed under the terms of the Creative Commons Attribution License, which permits unrestricted use, distribution, and reproduction in any medium, provided the original work is properly cited (http://creativecommons.org/licenses/by/4.0/deed.en).

Received: 10.03.2019

Accepted: 16.04.2019; Published: 30.04.2019 